\title{
Further studies on the use of Raman spectroscopy and X-ray diffraction for the characterization of TiC-containing carbon-carbon composites
}

\author{
A. Centeno, C. Blanco, R. Santamaría, M. Granda, R. Menéndez* \\ Instituto Nacional del Carbón, CSIC, Apdo. 73, 33080-Oviedo, Spain
}

\begin{abstract}
Raman spectroscopy and X-ray diffraction are used to study the crystalline structure of carbon-carbon and TiC-containing composites. The advantages and drawbacks of these techniques for the characterization of carbon-carbon composites are analyzed in the light of the distribution and arrangement of their components and the microstructural orientation of the supporting matrix. Analyses performed on longitudinal and transverse sections of the composites confirm that the measurements are affected by the orientation of the crystals. The overall crystalline parameters calculated by X-ray diffraction were unequivocally resolved for each single component by means of Raman spectroscopy. A significantly higher degree of order was observed in the TiC-containing matrix as a result of the catalytic graphitisation of the carbon achieved by the addition of titanium. In addition, Raman spectroscopy corroborated that the incorporation of $\mathrm{TiC}$ into the carbon matrix does not disrupt the orientation of the graphene planes of the matrix parallel to the fibre axis, a necessary characteristic for achieving an optimum heat transfer through the material.
\end{abstract}

\footnotetext{
* Corresponding author. Tel: +34 985119090. Fax.: + 34 985297662. E-mail address: rosmenen@incar.csic.es (Rosa Menéndez)
} 


\section{1.- INTRODUCTION}

There is increasing demand nowadays for materials of high thermal conductivity for applications in thermal management systems, such as heat sinks or plasma facing materials in fusion and fission devices. Carbon-carbon composites are potential candidates for such applications due to their excellent properties [1]. Thermal conductivity depends on the degree of structural order, which is strongly influenced by the processing conditions (i.e temperature, pressure), the precursor of the matrix, the type of fibre and its architecture [2, 3]. To ensure a good heat transfer through the carbon material, the graphene planes of the matrix must be oriented parallel to the fibre axis, which is affected by the above mentioned variables [1]. The addition of metals and metallic carbides, such as $\mathrm{TiC}, \mathrm{VC}$ or $\mathrm{ZrC}$, to the graphite precursors increases crystallinity due to their catalytic effect on graphitisation [4,5], and improves their thermal conductivity. However, this does not necessarily apply to carbon-carbon composites since the presence of particles may disturb the orientation of the matrix around the fibres and hinder optimum heat transfer [6].

The degree of structural order in carbon materials is usually related with the apparent size of the crystallites determined by X-ray diffraction (XRD) [7,8]. The $\mathrm{d}_{002}$ spacing and average crystallite size along the $a$ and $c$ axis $\left(\mathrm{L}_{\mathrm{a}}\right.$ and $\mathrm{L}_{\mathrm{c}}$ respectively) are useful indicators of the degree of graphitization of the carbon structure [8]. This technique has been extensively used to monitor the effects of thermal treatment on the carbon microstructure $[9,10]$. It has also served to study the evolution of graphitisation after the addition of dopants to the graphites [11]. Although XRD has also been used to characterize carboncarbon composites [12], the results are not as reliable, as the signals detected are affected by the different crystallinity of the components and the direction in which the crystals are oriented $[13,14]$. Two additional factors must be taken into account when interpreting the 
XRD results: (1) the sensitivity of the measurements to the components of a higher degree of order, even when quantitatively disordered fractions are predominant and (2) the proportion of each component exposed on the surface affect the diffraction pattern obtained. These drawbacks explain why some authors are reluctant to use XRD for the characterization of composites [13]. In recent years, Raman spectroscopy has been increasingly employed as an alternative to XRD to determine the crystallinity of carbon materials $[9,15-20]$. The variation in the position, width and intensity of the Raman bands, especially in the first order region (D, G, D' at 1350,1580 and $1620 \mathrm{~cm}^{-1}$ respectively), is used to determine the structural order of the material [20]. Compared to XRD, Raman spectroscopy offers a higher surface selectivity since the measurements can be performed on each individual component of the composite material (i.e matrix or fibre) $[21,22]$ and can be applied to the same component in different orientations [23].

In this paper Raman spectroscopy and XRD are used to study the crystalline structure of carbon-carbon composites and $\mathrm{TiC}$-containing carbon-carbon composites. Furthermore, the advantages and drawbacks of these techniques for characterizing these composites are analyzed in the light of the distribution and arrangement of the components and the microstructural orientation of the supporting matrix.

\section{2.- EXPERIMENTAL}

\section{1.-Carbon-carbon composites}

The composites under study were obtained using a 2D twill weave PAN-based carbon fibre preform as reinforcement. Mesophase pitch was used as the matrix precursor in one of the composites (CC). A second composite was obtained by adding TiC nanoparticles to the mesophase pitch precursor (Ti-CC). The total amount of Ti in the matrix of this composite was $\sim 13$ wt. $\%$. The materials had a volume fraction of fibres of the order of $60 \%$. Further 
details about the liquid impregnation process used for the preparation of these composites are provided elsewhere [24].

\section{2.- Characterization of carbon-carbon composites}

The microstructure of the composites was studied by means of polarised light optical microscopy using a Zeiss Axioplan microscope. The samples were embedded in resin, then ground with $\mathrm{SiC}$ of different grades and subsequently polished to $0.05 \mu \mathrm{m}$ with alumina. Both longitudinal and transverse sections of the composites were studied. Scanning electron microscopy (SEM) was also performed on the surfaces of the materials using a Zeiss DSM 942 microscope.

XRD of bulk composites was carried out employing a monochromatic $\mathrm{Cu} \mathrm{K} \alpha \mathrm{X}$-ray source in a D8 Advance Bruker diffractometer. The diffraction patterns were collected by step scanning using a step size setting of $0.02^{\circ}$ and a scan step time of $1 \mathrm{~s}$. The broadening of the diffraction peaks due to instrumental factors was corrected by means of a silicon standard. Up to three measurements for each material were performed on the longitudinal (parallel to the fibre laminates) and transverse (perpendicular to the fibre laminates) sections of the composites. Crystallinity parameters, lattice spacing in the c-direction $\left(\mathrm{d}_{002}\right)$ and apparent crystallite sizes along the $a$ and $c$ axes ( $\mathrm{L}_{\mathrm{a}}$ and $\mathrm{L}_{\mathrm{c}}$ respectively), were calculated using the Scherrer equation [25].

Raman spectra were obtained on a high resolution Jobin Yvon Horiba Raman microscope. Extended scans from 1000 to $3500 \mathrm{~cm}^{-1}$ were performed using typical exposure times of 5 s. Measurements on the components of the composites (fibre, mesophase-based matrix and resin-based matrix) were made using samples embedded in resin, ground with $\mathrm{SiC}$ of different grades and subsequently polished to $0.05 \mu \mathrm{m}$ with alumina. A microscope was 
employed to focus the excitation laser beam (green line of an argon laser $\lambda=532 \mathrm{~nm}$ ) onto the material. Up to 15 measurements were performed on each component. The intensity, position and width of the bands were determined using a mixed Gaussian-Lorentzian curvefitting procedure. The degree of disorder was estimated from the $I_{D} / I_{T}$ ratio, where $I_{D}$ corresponds to the intensity of the $\mathrm{D}$ band $\left(1350 \mathrm{~cm}^{-1}\right)$ and $\mathrm{I}_{\mathrm{T}}$ corresponds to the total intensity of all the first-order region bands (D, G, D' at 1350, 1580 and $1620 \mathrm{~cm}^{-1}$ respectively). The variation in the position, width and intensity of the Raman bands in the second-order region $\left(2200-3400 \mathrm{~cm}^{-1}\right)$ was also used to study the structural order. In this region the ordering of the materials was evaluated from the band at $2700 \mathrm{~cm}^{-1}$ ( $\mathrm{G}^{\prime}$ band), which splits into G1' and G2' for the more graphitised materials [26], the band at $3200 \mathrm{~cm}^{-1}$ (2D' band, a counterpart of the $\mathrm{D}^{\prime}$ band) and a third band at $\sim 2950 \mathrm{~cm}^{-1}\left(\mathrm{D}^{\prime \prime}\right.$, a combination of the $\mathrm{D}$ and $\mathrm{G}$ bands).

\section{3. - RESULTS AND DISCUSSION}

\subsection{Description of the carbon-carbon composites}

The composite materials studied consisted of 2D twill weave PAN-based carbon fibre laminates piled at $90^{\circ}$ and consolidated using resin. Optical microscopy images of longitudinal and transverse sections of the materials are shown in Figure 1. In the longitudinal section, the laminates are arranged perpendicularly and all the fibre bundles are arranged longitudinally (Figure 1a). In the transverse section (Figure 1b), corresponding to the laminates oriented at $90^{\circ}$, the longitudinal and cross-section of the fibres can be observed. 

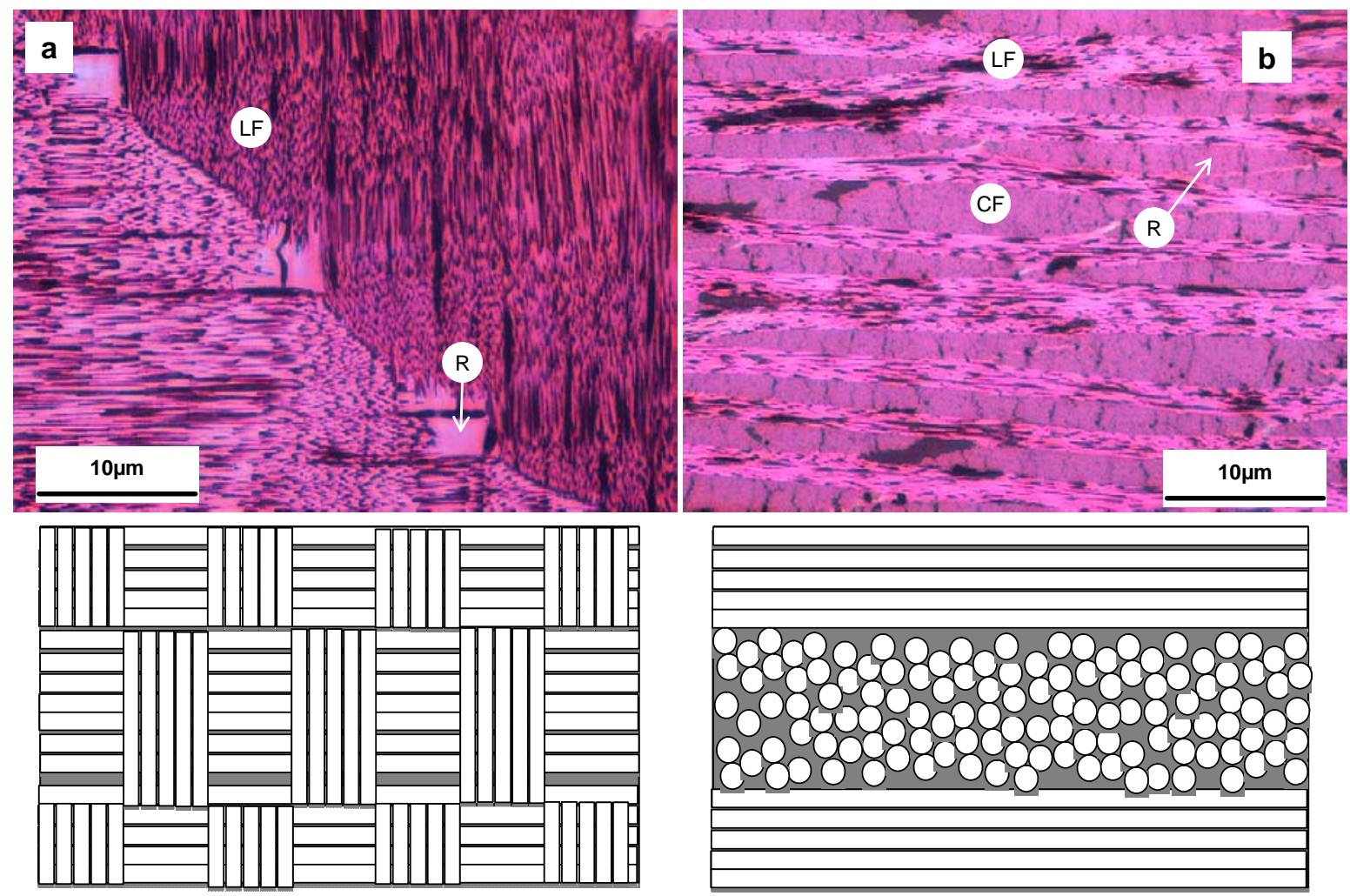

Figure 1.- Optical micrographs of composite $\mathrm{CC}$ and schemes of the fibre bundle arrangements: (a) longitudinal section and (b) transverse section. $L F$, longitudinal section of fibre; $C F$, cross-section of fibre and $R$, resin.

\subsection{Characterization of composites by XRD}

XRD performed on both the longitudinal and transverse sections of the bulk materials showed different diffraction patterns (Figure 2). As previously mentioned, in the longitudinal section the laminates are arranged perpendicularly to the incident diffraction beam and all the fibre bundles are arranged longitudinally (Figure 1a). In the transverse section (Figure $1 \mathrm{~b}$ ), both the longitudinal and cross-section of the fibres are exposed to the incident beam and contribute to the signal obtained. The diffraction pattern of the longitudinal section (Figure 2a) shows sharper peaks corresponding to the (001) reflection, 
which is characteristic of a three-dimensional order $\left(\mathrm{d}_{002}, \mathrm{~L}_{\mathrm{c}}\right)$ [27]. Meanwhile, the (hk0) reflections, which are characteristic of a two-dimensional order $\left(\mathrm{L}_{\mathrm{a}}\right)$, are diffuse and appear almost as a single band (hk). The resolution of these peaks changes when the transverse section is analysed (Figure $2 \mathrm{~b}$ ). In this case, the peaks corresponding to (hk0) are more sharply defined. The differences observed depending on the orientation of the material can be attributed to the distribution of the fibres in the reinforcement (Figure 1), and also to their crystalline structure. As PAN fibres consist of graphene planes oriented almost parallel to the fibre axis [28], the longitudinal and cross-sections of the fibres can be considered to approximately represent the basal and edge planes, respectively [29]. Additionally, the mesophase pitch tends to align with the graphene planes parallel to the fibre axis [30]. Therefore, when XRD is preformed on the longitudinal section of the material, it is assumed that the fibre and matrix will contribute to the three-dimensional order. In the case of the transverse section, the longitudinal and cross-section of the fibres and the matrix will contribute to both, the three-dimensional and the two-dimensional order.

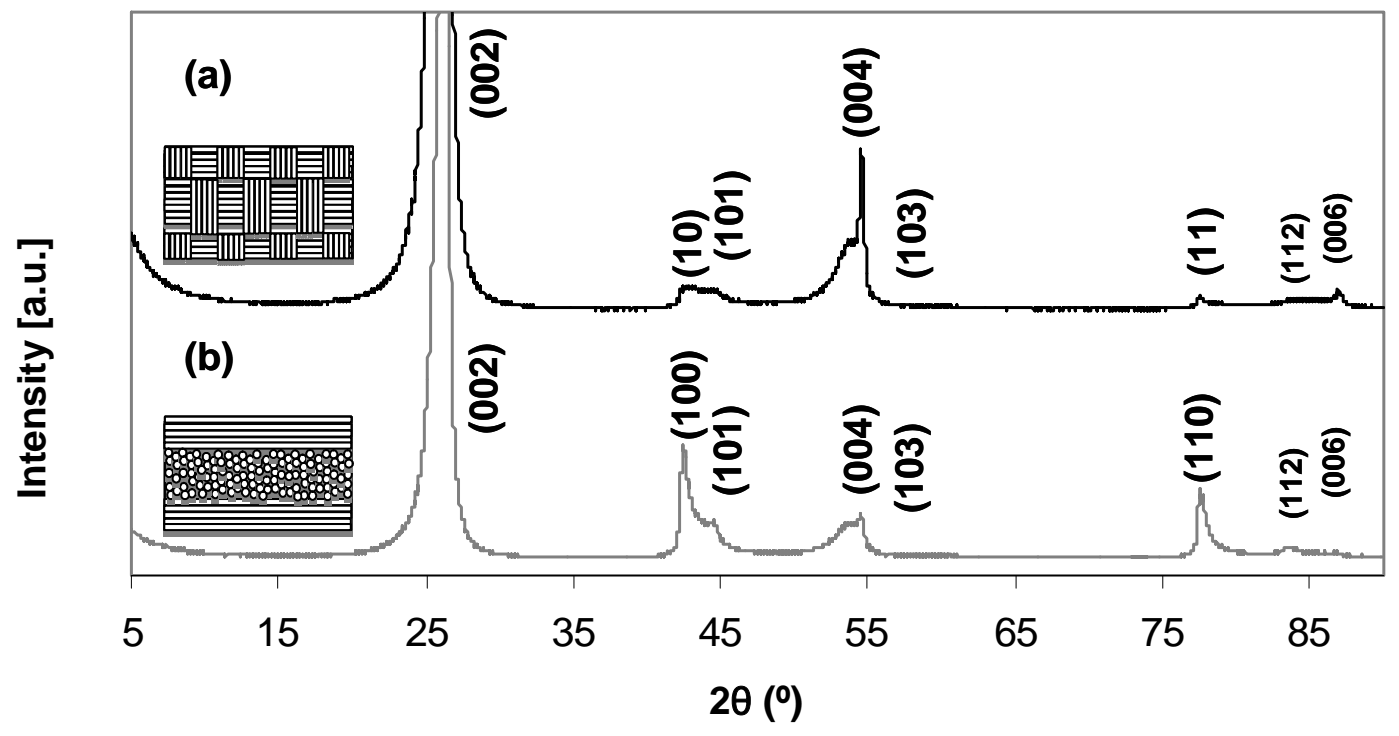

Figure 2.-X-ray diffraction patterns of composite CC: (a) longitudinal section and (b) transverse section. 
The qualitative changes observed in the diffraction patterns of the materials (heavily influenced by their orientation) were also accompanied by quantitative changes in the crystalline parameters (Table 1). For both composites, higher values of $\mathrm{L}_{\mathrm{a}}$ were obtained in the measurement of the transverse section, while higher values of $\mathrm{L}_{\mathrm{c}}$ were obtained in the case of the longitudinal section (Table 1). These differences are due to the different distribution of fibres and matrix in the composites and their different crystalline structure, as previously explained. In the transverse section there is a higher contribution of the basal planes of both matrix and fibres to the XRD signals associated to the two dimensional order [(100), (101)] (Figure 2) as a consequence of their distribution (Figure 1b). As a result, higher values of $\mathrm{L}_{\mathrm{a}}$ are obtained. Meanwhile, in the longitudinal section the beam lays perpendicular to the basal planes of both matrix and fibres (Figure 1b) and, therefore, there is a higher contribution of the signals associated to the three dimensional order (002), reflected in higher values of Lc in this section.

The higher values of $\mathrm{L}_{c}$ and $\mathrm{L}_{\mathrm{a}}$ obtained for Ti-CC, in both orientations, show that the presence of $\mathrm{TiC}$ contributes to the development of a more ordered matrix. The higher differences in the crystalline parameters of $\mathrm{CC}$ and Ti-CC composites are especially evident in the case of the longitudinal section. These higher values of crystalline parameters may be related to the graphene planes of the matrix being oriented parallel to the fibre axis [30], the highest crystalline order corresponding to the basal planes of the matrix. Though in a lower proportion compared to the fibres ( 22 vs $60 \%$ vol.), the matrix is the component that makes the highest contribution to the values obtained by XRD due to its higher degree of order. 
Table 1.-XRD crystalline parameters.

\begin{tabular}{lcccc}
\hline & COMPOSITE & $\begin{array}{c}\mathrm{d}_{002} \\
(\AA)\end{array}$ & $\begin{array}{c}\mathrm{L}_{\mathrm{a}} \\
(\AA)\end{array}$ & $\begin{array}{c}\mathrm{L}_{\mathrm{c}} \\
(\AA)\end{array}$ \\
\hline Longitudinal section & CC & 3.370 & 512 & 424 \\
& Ti-CC & 3.368 & 570 & 460 \\
Transverse section & CC & 3.372 & 595 & 416 \\
& & & & \\
& Ti-CC & 3.370 & 605 & 440 \\
\hline
\end{tabular}

3.3 Characterization of composites by Raman spectroscopy

By means of Raman spectroscopy it is possible to identify the degree of order of each single component on the basis of the spectra and the intensity ratio of the D band (which is associated with some type of structural disorder [20]) and the G band (typical of the Raman spectrum of graphite [31]). The largest qualitative differences are observed in the first-order region. If the transverse section of the material is analysed, the lowest D intensity corresponds to that exhibited by the cross-section of the matrix and the longitudinal section of the fibre (Figure 3). These components exhibit the lowest $\mathrm{I}_{\mathrm{D}} / \mathrm{I}_{\mathrm{T}}$ ratio, especially in the case of the Ti-CC matrix (Table 2), as a result of the catalytic activity promoted by titanium which improves the degree of order. 


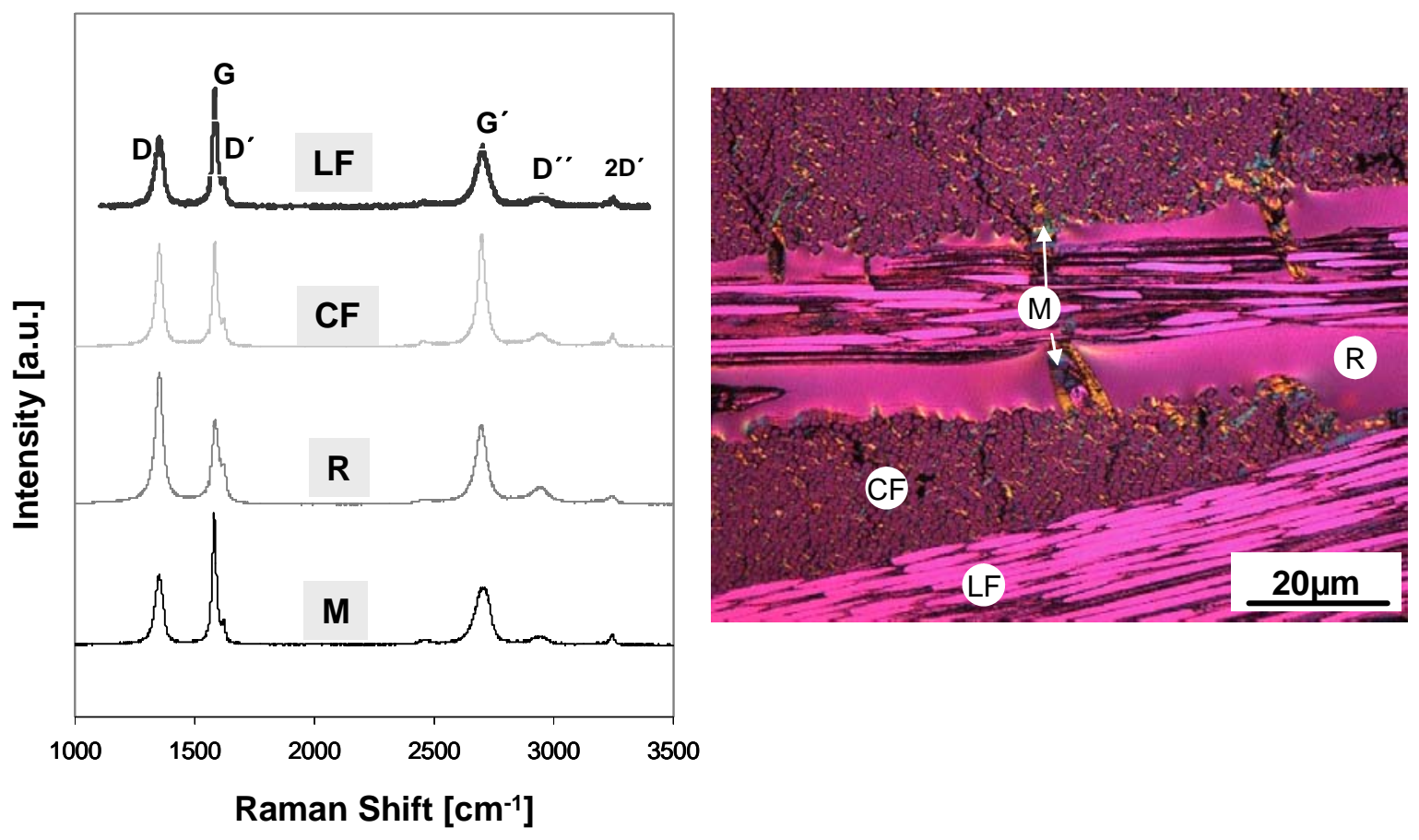

Figure 3.-Raman spectra of individual components and optical micrograph of the transverse section of composite CC. LF, longitudinal section of fibres; $R$, resin; $C F$, crosssection of fibres and $M$, cross-section of matrix.

Table 2.-Raman parameters obtained from the transverse section of the composites.

\begin{tabular}{|c|c|c|c|c|c|c|c|c|c|}
\hline Component & $v_{G}$ & $\mathrm{FWHM}_{\mathrm{G}}$ & $\mathbf{I}_{\mathbf{D}} / \mathbf{I}_{\mathbf{T}}$ & $v_{G^{\prime}}$ & FWHM $_{\mathrm{G}^{\prime}}$ & $v_{D^{\prime \prime}}$ & FWHM $_{\text {D" }}$ & $\mathbf{v}_{2 \mathrm{D}}$ & $\mathrm{FWHM}_{2 \mathrm{D}}$ \\
\hline $\begin{array}{l}\text { CC matrix } \\
\text { (c.s.) }\end{array}$ & 1581 & 20 & 50 & 2701 & 65 & 2940 & 58 & 3244 & 17 \\
\hline $\begin{array}{l}\text { Ti-CC } \\
\text { matrix } \\
\text { (c.s.) }\end{array}$ & 1580 & 20 & 39 & 2702 & 62 & 2940 & 55 & 3244 & 13 \\
\hline Fibre (c.s.) & 1582 & 22 & 56 & 2698 & 42 & 2941 & 60 & 3242 & 25 \\
\hline Fibre (1.s.) & 1581 & 21 & 46 & 2701 & 58 & 2940 & 58 & 3242 & 23 \\
\hline Resin & 1584 & 31 & 63 & 2696 & 51 & 2941 & 65 & 3239 & 40 \\
\hline \multicolumn{10}{|c|}{ 1.s.: Longitudinal section } \\
\hline$v$ : Band posi & ion $(\mathrm{cr}$ & & WHM & Full w & dth at half- & $\operatorname{maxim}$ & ln $\left(\mathrm{cm}^{-1}\right)$ & & \\
\hline
\end{tabular}


If the fibre orientations are compared, the intensity of the $\mathrm{D}$ band is lower in the longitudinal section than in the cross-section, the $\mathrm{I}_{\mathrm{D}} / \mathrm{I}_{\mathrm{T}}$ ratios being of 46 and 56 , respectively. Since the degree of graphitization should be similar throughout the section of the fibre, the differences between the spectra must be due to the orientation of the graphene planes (the longitudinal and cross-sections may be considered as being roughly similar to basal and edge planes, respectively).

Although the second-order Raman spectra are not so commonly studied as the first-order ones, this region is often more sensitive to graphite ordering. In our case, all the components show well defined bands (Figure 3) that are typical of well-ordered materials. The shift of the $\mathrm{G}^{\prime}\left(\sim 2700 \mathrm{~cm}^{-1}\right)$ and $2 \mathrm{D}^{\prime}\left(\sim 3254 \mathrm{~cm}^{-1}\right)$ bands to higher wave numbers and the narrower width at half maximum observed in the Ti-CC matrix, compared to that of $\mathrm{CC}$, indicate that this material has a more graphitic structure [32].

\subsubsection{Degree of structural order corresponding to different matrix orientations}

The results shown so far demonstrate that the incorporation of $\mathrm{TiC}$ into the matrix produces changes in the crystallinity. Therefore, it is of especial interest to study the crystallinity of the matrix under different orientations. Unpolished specimens of the longitudinal section of the composite with the matrix deposited on the fibres were used for this purpose (Figure $4 b)$. 


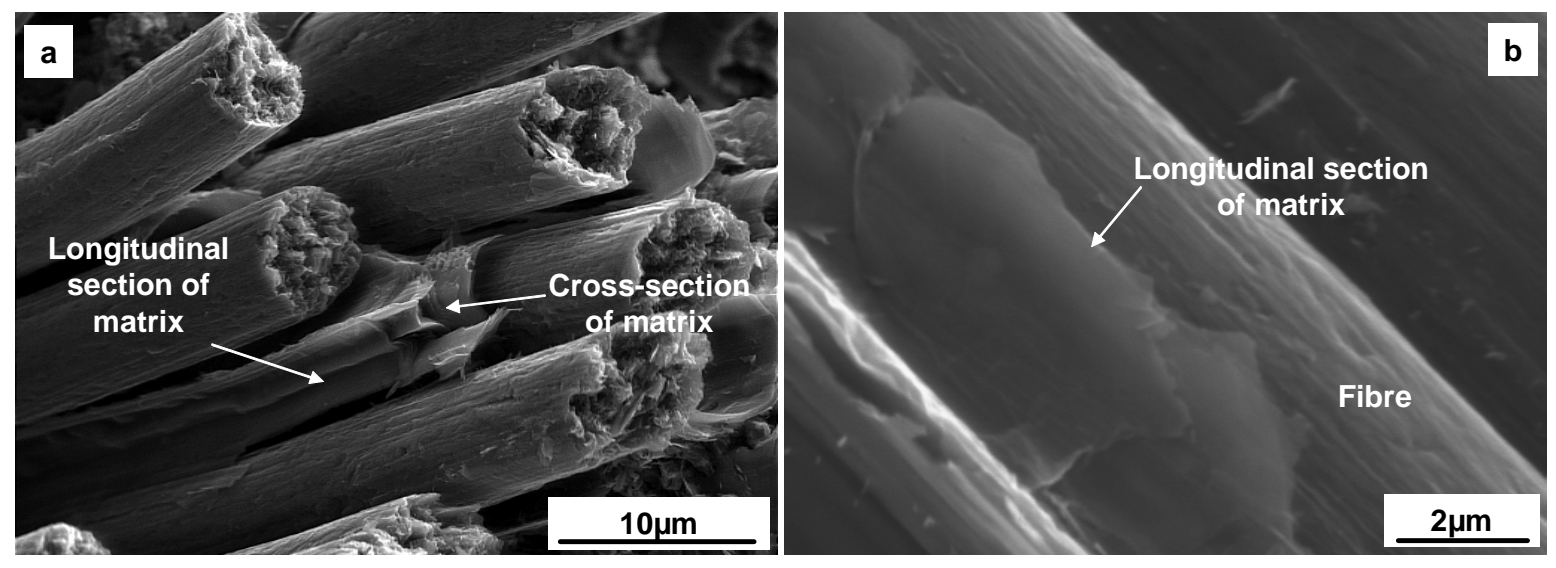

Figure 4.- SEM images of composite CC.

The Raman spectra of the longitudinal section of the matrix (Figure 5) reveal significant differences when compared to those obtained from the cross-section (Figure 3). In the firstorder region, there is a marked increase in the intensity of the $G$ band and the width decreases with a shift towards a lower wavenumber as it approaches the value of graphite $\left(1580 \mathrm{~cm}^{-1}\right)$. The intensity of the $\mathrm{D}$ band decreases significantly until it almost disappears, especially in the Ti-CC matrix, while D' (which according to Katagiri et al. [33] is typical of the spectrum of plane edges of graphite), disappears completely, verifying that the analysis corresponds to the basal planes of the matrix. These results confirm that the planes of the matrix are oriented parallel to the fibre axis, as was previously inferred from the XRD analysis. The $\mathrm{I}_{\mathrm{D}} / \mathrm{I}_{\mathrm{T}}$ ratio of the longitudinal section is noticeably lower than that corresponding to the cross-section, with values of 4 and 50 for the $\mathrm{CC}$ matrix, indicating that there is a more highly oriented structure around the fibres. In comparison with the cross-section of the matrix (Figure 3), the second-order region of the longitudinal section does not show a D" band $\left(\sim 2900 \mathrm{~cm}^{-1}\right)$ that is associated with defects, and $\mathrm{G}^{\prime} \sim 2700 \mathrm{~cm}^{-1}$ is highly asymmetric. These characteristics, typical of the basal planes of graphite, confirm beyond doubt that the matrix is highly graphitic, the basal planes being highly oriented along the fibre axis. 


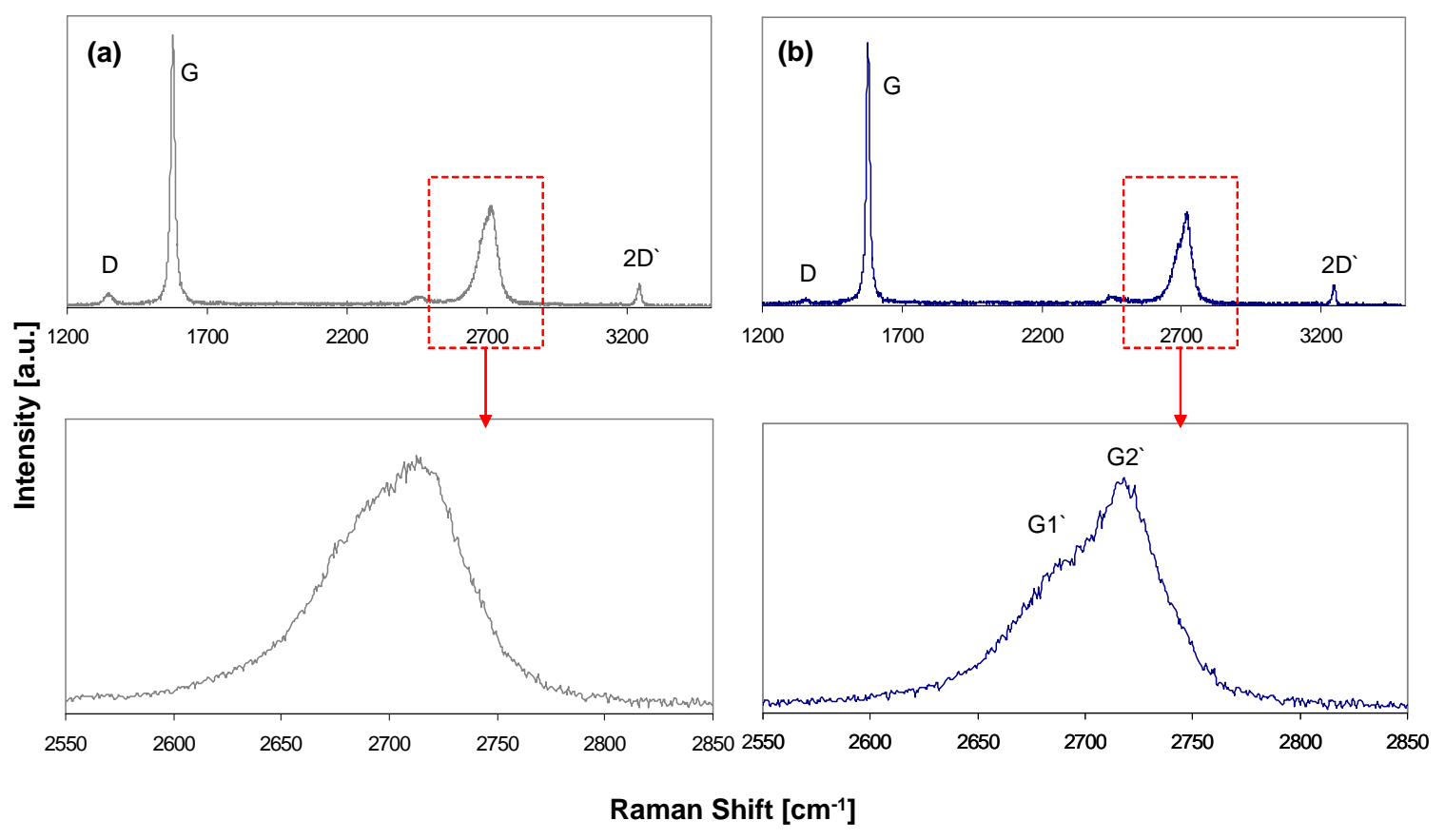

Figure 5.- Raman spectra of the longitudinal section of unpolished matrix: (a) CC and (b) Ti-CC.

The changes in crystallinity due to the incorporation of $\mathrm{TiC}$ are especially remarkable in the longitudinal section (Figure 5). In the first-order region of the Ti-CC matrix (Figure 5b), the $\mathrm{D}$ band is almost inappreciable, which is indicative of the high structural order of the matrix in this direction, reflected also in the $\mathrm{I}_{\mathrm{D}} / \mathrm{I}_{\mathrm{T}}$ ratio $(1$ and 4 for Ti-CC and $\mathrm{CC}$ matrix respectively). In the second-order spectrum of the Ti-CC matrix, the $\mathrm{G}^{\prime}$ band at $2700 \mathrm{~cm}^{-1}$ splits into G1' and G2' which is typical of most graphitised materials [26]. These features are related with the final stage of graphitization, as Lespade et al. have pointed out [34] and reflect the higher degree of graphitization reached after the incorporation of $\mathrm{TiC}$ into the matrix due to its catalytic effect. 


\section{4.- CONCLUSIONS}

The analysis of longitudinal and transverse sections of composites provides significant additional information to complement the results obtained by XRD and Raman spectroscopy.

XRD has demonstrated the influence of the orientation of the material on the diffraction patterns obtained and consequently on the crystalline parameters. XRD measurements are highly sensitive to components with a higher degree of order and the proportion of each component exposed on the surface measured affects the diffraction pattern obtained.

Analysis of the individual components by means of Raman spectroscopy has evidenced that the matrix is high oriented parallel to the axis of the fibre not only in the case of $\mathrm{CC}$ but also in Ti-CC, thereby demonstrating that the incorporation of $\mathrm{TiC}$ does not disturb the orientation of the matrix around the fibres.

Evaluation of the second order Raman spectra corresponding to the matrix under both orientations (along and perpendicular to the fibre axis), confirms that the graphene planes are arranged parallel to the fibre axis and highly oriented along the fibre axis, together with a highly graphitic structure of the matrix, especially in the presence of TiC.

Acknowledgements.- This work has been performed within the framework of the Integrated European Project "ExtreMat" (contract NMP-CT-2004-500253) with financial support from the European Community and the Spanish Education Ministry (MAT200422787-E). 


\section{REFERENCES}

[1] Savage G. Carbon-carbon composites. London: Chapman and Hall; 1993.

[2] Luo R, Liu T, Li J, Zhang H, Chen Z, Tian G. Thermophysical properties of carbon/carbon composites and physical mechanism of thermal expansion and thermal conductivity. Carbon 2004; 42(14): 2887-95.

[3] Jie C, Xiang X, Peng X. Thermal conductivity of unidirectional carbon/carbon composites with different carbon matrixes. Materials \& Design 2009; 30(4): 1413-6.

[4] Qiu H, Song Y, Liu L, Zhai G, Shi J. Thermal conductivity and microstructure of Tidoped graphite. Carbon 2003; 41(5): 973-8.

[5] Liu Z, Guo Q, Shi J, Zhai G, Liu L. Preparation of doped graphite with high thermal conductivity by a liquid mixing process. Carbon 2007; 45(9): 1914-6.

[6] Manocha LM, Warrier A, Manocha S, Sathiyamoorthy D, Banerjee S. Thermophysical properties of densified pitch based carbon/carbon materials - I. Unidirectional composites Carbon 2006; 44: 480-7.

[7] Franklin RE. Crystallite growth in graphitizing and non-graphitizing carbons.

Proceedings of the Royal Society of London. Series A, Mathematical and Physical Sciences 1951; 209: 196-218.

[8] Pierson HO. Handbook of carbon, graphite, diamond and fullerenes: properties, processing, and applications. Park Ridge, NJ: Noyes Publications; 1993.

[9] Zickler GA, Smarsly B, Gierlinger N, Peterlik H, Paris O. A reconsideration of the relationship between the crystallite size La of carbons determined by X-ray diffraction and Raman spectroscopy. Carbon 2006; 44(15): 3239-46. 
[10] Nadeau G, Song XY, Massé M, Guerfi A, Brisard G, Kinoshita K, et al. Effect of heattreatment and additives on the particles and carbon fibers as anodes for lithium-ion batteries Journal of Power Sources 2002; 108, 1-2(1): 86-96.

[11] García-Rosales C, Ordás N, Oyarzabal E, Echeberria J, Balden M, Lindig S, et al. Improvement of the thermo-mechanical properties of fine grain graphite by doping with different carbides Journal of Nuclear Materials 2002; 307-311(2): 1282-8.

[12] Wu X, Radovic LR. Inhibition of catalytic oxidation of carbon/carbon composites by boron-doping Carbon 2005; 43(8): 1768-77.

[13] Thrower PA. Editorial. Carbon 2004; 42: 699

[14] Dobiášová L, Starý V, Glogar P, Valvoda V. Analysis of carbon fibers and carbon composites by asymmetric X-ray diffraction technique Carbon 1999; 37(3): 421-5.

[15] Cuesta A, Dhamelincourt P, Laureyns J, Martínez-Alonso A, Tascón JMD.

Comparative performance of X-ray diffraction and Raman microprobe techniques for the study of carbon materials. Journal of Materials Chemistry 1998; 8: 2875-9.

[16] Tuinstra F, Koenig JL. Raman spectrum of graphite. Journal of Chemical Physics 1970; 53: 1126-30.

[17] Nakamizo M, Kammereck R, Walker PL Jr. Laser Raman studies on carbons. Carbon $1974 ; 12: 259-67$.

[18] Sadezky A, Muckenhuber H, Grothe H, Niessner R, Pöschl U. Raman microspectroscopy of soot and related carbonaceous materials: spectral analysis and structural information. Carbon 2005; 43: 1731-42.

[19] Beyssac O, Goffé B, Petitet JP, Froigneux E, Moreau M, Rouzaud JN. On the characterization of disordered and heterogeneous carbonaceous materials by Raman spectroscopy. Spectrochimica Acta Part A 2003; 59(10): 2267-76. 
[20] Ferrari AC, Robertson J. Interpretation of Raman spectra of disordered and amorphous carbon. Physical Review. B 2000; 61(20): 14095-107.

[21] Linhua Z, Baiyun H, Yong H, Qizhong H, Chang'an W. An investigation of heterogeneity of the degree of graphitization in carbon-carbon composites. Materials Chemistry and Physics 2003; 82(3): 654-62.

[22] Hamada K, Sato S, Kohyama A. Proceedings of the Ninth International Conference on Composite Materials (ICCM/9). Department of Mechanical Engineering, University of Zaragoza (Madrid, Spain), 1993, p. 516.

[23] Endo M, Kim C, Karaki T, Tamaki T, Nishimura Y, Matthews MJ, et al. Structural analysis of the B-doped mesophase pitch-based graphite fibres by Raman Spectroscopy. Physical Review B 1998; 58(14): 8991-6.

[24] Centeno A,. Santamaría R, Granda M, Menéndez R, Blanco C. Improvement of thermal conductivity in 2D carbon-carbon composites by doping with TiC nanoparticles Materials Chemistry and Physics 2010; 122(1): 102-7.

[25] Warren BE, Biscoe JH. An x-ray study of carbon black. Journal of Applied Physics 1942; 13(6): 364-71.

[26] Ferrari AC, Meyer JC, Scardaci V, Casiraghi C, Lazzeri M, Mauri F, et al. Raman spectrum of graphene and graphene layers. Physical Review Letters 2006; 97(18) 187401. [27] Iwashita N, Park C R, Fujimoto H, Shiraishi M, Inagaki M. Specification for a standard procedure of X-ray diffraction measurements on carbon materials. Carbon 2004; 42(4): 701-14.

[28] Fitzer E, Manocha LM. Carbon reinforcements and carbon/carbon composites. Berlin: Springer-Verlag, 1998, p. 3-66. 
[29] Montes-Morán M A, Young RJ. Raman spectroscopy study of HM carbon fibres:

effect of plasma treatment on the interfacial properties of single fibre/epoxy composites:

Part I: Fibre characterisation. Carbon 2002: 40(6): 845-55.

[30] White JL, Sheaffer PM. Pitch-based processing of carbon-carbon composites. Carbon 1989; 27(5): 697-707.

[31] Vidano R, Fischbach DB. New lines in the Raman spectra of carbons and graphite. J Am Ceram Soc 1978; 61: 13-7.

[32] Hishiyama Y, Irumano H, Kaburagi Y, Soneda Y. Structure, Raman scattering, and transport properties of boron-doped graphite Phys. Rev. B 2001; 63: 245406.

[33] Katagiri G, Ishida H, Ishitani A. Raman spectra of graphite edge planes. Carbon 1988; 26(4): 565-71.

[34] Lespade P, Marchand A, Couzi M, Cruege F. Caracterisation de materiaux carbones par microspectrometrie Raman. Carbon 1984; 22(4-5): 375-85. 